\title{
Comparing genomic-based estimates of relatedness for use in pedigree-based conservation management
}

4 Samantha Hauser ${ }^{1}$, Stephanie J Galla ${ }^{2.3}$, Tammy E Steeves ${ }^{2}$, Emily K Latch ${ }^{1}$

5 'Department of Biological Sciences, University of Wisconsin - Milwaukee, USA

$6 \quad{ }^{2}$ School of Biological Sciences, University of Canterbury, New Zealand

7 Department of Biological Sciences, Boise State University, USA

9 Running head: genomic estimates of relatedness

11 ORCiD Hauser: 0000-0002-3404-5253

12 ORCiD Galla: 0000-0002-4650-8067

13 ORCiD Steeves: 0000-0003-2112-5761

14 ORCiD Latch: 0000-0002-9892-1056

15

Keywords: relatedness, allele-sharing, captive breeding, ex situ management, conservation

17 breeding program, wild pedigrees

18

19 


\section{Abstract}

1. Researchers have long debated which genomic estimator of relatedness best captures the degree of relationship between two individuals. In the genomics era, this debate continues, with relatedness estimates being sensitive to the method used to generate genomic markers (e.g., reduced-representation sequencing, whole genome resequencing), marker quality, and levels of diversity in sampled individuals.

2. Here, we compare six commonly used relatedness estimators (kinship genetic distance (KGD), Wang Maximum Likelihood (TrioML), Queller and Goodnight $\left(\mathrm{R}_{\mathrm{xy}}\right)$, KINGrobust, $\mathrm{R}_{\mathrm{AB}}$, allele-sharing co-ancestry) across five species bred in captivity-including three birds and two mammals-with varying degrees of reliable pedigree data, using reduced-representation and whole genome resequencing data.

3. Relatedness estimates varied widely across estimators, sequencing method, and species, yet the most consistent results with known pedigree data were found using KING-robust and to a lesser extent KGD. The allele-sharing estimator was sensitive to missing data and inbreeding, attributes that make this estimator ill-suited for use in captive breeding programs. Our combined results indicate there is not a single genomic based estimator that is ideal across different species and data types.

4. To enable researchers to evaluate the most appropriate relatedness estimator for each new data set, we provide a structured workflow that is broadly applicable to conservation breeding programs, particularly where genomic estimates of relatedness can complement and complete poorly pedigreed populations. Given a growing interest in wild pedigrees, our results and workflow are also applicable to in situ wildlife management. 


\section{Introduction}

Relatedness and kinship, concepts that quantify the relationship between two individuals

45 (hereafter referred to relatedness in the general sense), are foundational in biology (Wright,

46 1922), with application in health sciences (Ott, 1974), agriculture (Cassell et al., 2003; Jannink et

47 al., 2001) and species conservation (Fernández et al., 2005). Pedigrees track relatedness in a

48 population by documenting the ancestry of individuals and have been an important tool for

49 conservation and management of populations. In a conservation context, pedigrees have been

50 fundamental to the management of small populations, including those in captivity (i.e., ex situ

51 population management) or intensively managed wild or semi-wild populations (i.e., in situ or

52 “sorta situ”; Wildt et al., 2019; Wolfe et al., 2012). Practitioners can prioritize individuals with

53 low mean kinship when making pairing decisions in captivity, or population management

54 decisions in wild or semi-wild populations (Ballou \& Lacy, 1995; Giglio et al., 2016; Weeks et

55 al., 2011). A management strategy that minimizes mean kinship in a population is effective at

56 mitigating drift, inbreeding, and adaptation to captivity, while preserving genetic diversity and

57 evolutionary potential in an effort to curtail extinction risk (Fernandez \& Toro, 1999;

58 Montgomery et al., 1997; Sonesson \& Meuwissen, 2001; Spielman, 2004). Simulation studies

59 have shown that pedigree-based management of small populations minimizes short-term

60 inbreeding while maximizing founder diversity (e.g., Ballou \& Lacy, 1995; Rudnick \& Lacy,

61 2008). Empirical studies have demonstrated the importance of pedigree management for

62 threatened species conservation (e.g., Tasmanian devil, Sarcophilus harrisii, Gooley et al, 2017;

63 Ālala, Corvus hawaiiensis, Flanagan et al., 2021; Bison, Bison bison, Giglio et al., 2018).

64 Because the collection of pedigree data is feasible for many captive and intensively managed

65 wild populations and computer programs are readily available for studbook management (e.g., 
66 PopLink, ZIMS; Faust et al., 2019, Species360, pedigree analysis (e.g., PMx; Lacy et al., 2012),

67 and population modeling (e.g., VORTEX; Lacy \& Pollak, 2014), pedigree-based genetic

68 management of populations is an accessible and appealing tool for genetic management.

69 Pedigrees continue to provide high precision estimates of inbreeding and relatedness

70 when pedigrees are robust (i.e., many generations deep with relatively low missing data; Putnam

71 \& Ivy, 2014; Robinson et al., 2013). However, there are several limitations common in pedigrees

72 for small populations that can hinder their utility for genetic management (Galla et al. Preprint).

73 One is that the individuals forming the initial population (hereafter, founders) are often of

74 unknown relationships and assumed to be equally unrelated (Ballou 1983; Hogg et al., 2019). In

75 small populations that have experienced sustained bottlenecks, it is unlikely that remaining

76 individuals are unrelated. For example, a genetic study of critically-endangered kākāpō (Strigops

77 habroptilus) revealed high relatedness coefficients — including some first-order (i.e., parent-

78 offspring or sibling) relationships — among founders that were previously assumed to be

79 unrelated (Bergner et al., 2014). Assuming founders are unrelated can lead to underestimated

80 kinship and inbreeding coefficients, which is exacerbated when pedigrees are shallow $(<5$

81 generations deep; Balloux et al., 2004 but see Rudnick and Lacy 2008). Beyond the initial

82 founding event, additional founders can be incorporated into pedigrees when populations are

83 augmented by wild individuals with unknown relationships to each other or to individuals

84 already in the pedigreed population (Spielman \& Frankham (Galla et al., 2020; Spielman \&

85 Frankham, 1992).

86 Pedigree gaps indicating unknown relationships can also be a source of ambiguity

87 constraining the utility of pedigrees for genetic management. Missing data can arise from

88 uncertainties in parentage, for example through undetected extra-pair parentage or herd or 
89 colonial breeding systems (Overbeek et al., 2020; Ferrie, 2017; Mucha \& Windig, 2009). In the

90 wild, pedigree gaps may be introduced or relationships incorrectly inferred due to individual

91 identification errors (e.g., dropped leg bands; Milligan et al., 2003). Parentage assignment errors

92 may be rare (Henkel et al. 2012, Ferrie et al. 2013, Hammerly et al. 2016) but their effects can

93 compound over generations, increasing the probability that related individuals will be

94 unintentionally paired and negatively impacting population fitness. For example, in a captive

95 population of Attwater's Prairie-chicken (Tympanuchus cupido attwateri), nearly $40 \%$ of the

96 population were direct descendants of only 4 errors 4 years prior (Hammerly et al., 2016). To

97 alleviate pedigree shortcomings, including unknown founder relationships, missing pedigree

98 data, and potential error, researchers can use genomic estimates of relatedness to complement

99 and complete pedigrees.

Relatedness estimates generated using genomic markers have helped to resolve founder

101 relationships (Bergner et al., 2014), reconstruct parentage (Flanagan \& Jones, 2019), and vet

102 pedigrees for potential errors (Hammerly et al., 2016). Though, factors that can affect the

103 accuracy of genetically-estimated relatedness include marker type, number of loci, and the

104 specific estimator used. Single nucleotide polymorphisms (SNPs), which can be derived from

105 SNP-assays and arrays (Santure et al., 2010), reduced representation sequencing (RRS)

106 approaches like RAD-sequencing or GBS (Galla et al., 2019; Lemopoulos et al., 2019), or whole

107 genome resequencing (WGS) approaches (Galla et al., 2020), yield accurate genomic estimates

108 of relatedness (Jones \& Wang, 2010; Santure et al., 2010b; Skare et al., 2009). But, the quantity

109 of markers necessary for a given system depends upon the genome size of the species, population

110 size, and overall level of inbreeding in the population (Morin et al., 2009; Smouse, 2010; Sun et

111 al., 2016); insufficient markers can provide inaccurate relationship inference. Similarly, the 
112 specific estimator used to capture the inheritance patterns and translate them into a relatedness

113 estimate can impact the accuracy of relationship inference.

114 There are several commonly used methods to estimate genomic-based relatedness and

115 reconstruct pedigrees, each with their own limitations. Frequency-based estimates of relatedness

116 quantify the probability that shared alleles are identical-by-descent (IBD) relative to a reference

117 population using probabilities (i.e., moment-methods) or correlations (likelihood-based; Wang,

118 2014). Frequency-based estimators assume that populations are large, randomly mating, and

119 outbred (but see (Hedrick \& Lacy, 2015; Wang, 2007), effectively sampled (Wang 2017), and

120 that allele frequencies at each marker are reliably estimated (Csillery et al. 2006, Galla et al.

121 2020). However, there are notable exceptions: the KGD estimator (Dodds et al., 2015) uses only

122 pairs of individuals and therefore quantifies identity-by-state rather than IBD; $\mathrm{R}_{\mathrm{AB}}$ (Korneliussen

123 \& Moltke, 2015) and KING-robust (Waples et al., 2019) account for unrealistic assumptions

124 such as a large panmictic population. A meaningful reference population is difficult to define for

125 captive populations, where individuals are managed through non-random mating strategies and

126 often divided across numerous institutions. Further, the precision of frequency-based approaches

127 will rely on marker completeness and richness, with more markers leading to more precise

128 estimates of relatedness (Galla et al., 2020). As an alternative, pairwise allele sharing (i.e.,

129 molecular co-ancestry or similarity index; Gutiérrez et al., 2005) does not rely upon reference

130 population allele frequencies and has been found to be strongly correlated with mean kinships

131 derived from pedigrees, thus making it a potentially useful relatedness estimator for populations

132 where the assumptions of frequency-based estimators are violated, such as captive populations

133 (Ivy et al., 2016). 
In this study, we compared the accuracy of six genomic-based estimators relative to

135 observed pedigree data, including allele-sharing and five different frequency-based relatedness

136 estimators across 11 genomic datasets. Best practice guidance to estimate accurate genomic-

137 based relatedness using high throughput sequencing data is needed to maximize conservation

138 success for poorly-pedigreed and threatened populations. Relatedness estimates are derived from

139 SNP data generated using three different approaches (WGS and RRS with high and low levels of

140 missing data), collected from five different species with ex-situ breeding programs, including

141 Addax (Addax nasomaculatus), Inca Tern (Larosterna inca), Koala (Phascolarctos cinereus),

142 Kakī (Black Stilt; Himantopus novaezelandiae), and Kākāriki Karaka (Orange-fronted Parakeet;

143 Cyanoramphus malherbi). These five species offer a variety of realistic challenges in breeding

144 programs, including the size of the managed population, depth of the pedigree, and the amount

145 and structure of pedigree gaps (Figure 1). Imperfect pedigrees, as featured in the present study

146 and common in small populations, expose limitations of existing relatedness estimators.

147 Evaluation and identification of optimal relatedness estimators for use in conseration breeding

148 programs will improve pairing recommendations, maximize genetic diversity and in turn

149 increase species persistence; all critical goals of ex-situ conservation. Beyond broad applicability

150 to conservation breeding programmes, use of genetically-derived wild pedigrees (Pemberton,

1512008 ) will benefit from explicit evaluation of genomic estimators.

\section{Methods}

153 We used five species with conservation breeding programs: Addax, Inca Tern, Koala,

154 Kak̄̄ and Kākāriki Karaka in the present study. The Addax, Inca Tern and Koala study

155 populations are currently managed by the Association of Zoos and Aquariums (AZA) Population

156 Management Center (USA), and Kakī and Kārāriki Karaka are managed by the New Zealand 
157 Department of Conservation. These species represent a variety of realistic scenarios and

158 challenges practitioners face when generating breeding recommendations (Table 1; e.g.,

159 unknown founder relationships, gaps in pedigrees, inbreeding). The Addax pedigree was

160 historically not well-maintained; record keeping of parentage is challenging in herd managed

161 species, and these uncertainties compound over the pedigree. In addition, a large portion of the

162 AZA population originated from the private sector, adding to the uncertainty in the pedigree.

163 Only $13 \%$ of the AZA Addax pedigree is known. The contemporary pedigree for the Koala is

164 well known, but unknown relationships among founders and between contemporary imported

165 individuals and the captive population add gaps in the pedigree. Currently, 39\% of the Koala

166 pedigree is known. The Inca Tern pedigree is 30\% known, with unknown ancestry coming from

167 contemporary individuals that are managed in colonies. The Kakī pedigree has been maintained

168 since the early 1980's and includes intensively managed wild and captive individuals. About half

169 of the Kakī pedigree is known (56.6\%); pedigree gaps come from unbanded birds (gaps) and

170 extra-pair copulation (errors) in the wild (Overbeek et al., 2020). The Kākāriki Karaka pedigree

171 is a nearly complete pedigree, with $99.5 \%$ of the pedigree known. While complete, this pedigree

172 is shallow (0-3 generations deep), and as a result may be more impacted by incorrect

173 assumptions about founder relationships.

174 We generated SNP data for each of the five species using WGS and two common RRS

175 apparoches (double-digest restriction-site associated DNA (ddRAD) and genotype-by-

176 sequencing (GBS): ddRAD sequencing data for the Addax, Inca Tern, and Koala, GBS data for

177 Kakī (Galla et al., 2019), and WGS from Kakī and Kākāriki Karaka (Galla et al., 2020). SNP

178 data from WGS and GBS data for Kakī and Kākāriki Karaka were processed, and genotypes

179 were called as per Galla et al $(2019,2020)$. Briefly, Kakī GBS data was generated from two 
180 batches of individuals, Illumina Hi-Seq reads underwent reference-guided SNP discovery using

181 Tassel 5.0 (Glaubitz et al., 2014), and biallelic SNPs were filtered for a minimum MAF of 0.05,

182 a minimum SNP-depth of five, maximum of $10 \%$ missing data, and linkage disequilibrium $\left(r^{2}=\right.$

1830.6 over 1000 sites; Galla et al. 2019). For Kakī and Kākāriki Karaka WGS, reads were

184 generated from Illumina Hi-Seq or NovaSeq platforms, SNPs were discovered using BCFtools v.

185 1.9 (Li et al., 2009), and biallelic SNPs were filtered for a minimum MAF of 0.05, a quality

186 score greater than 20, a maximum of $10 \%$ missing data, linkage disequilibrium $\left(r^{2}=0.6\right.$ over

1871000 sites), and either a minimum depth of 5 or a minimum average depth of 10 , depending on

188 the species (Galla et al. 2020).

The ddRAD library preparation, quality control, and sequencing were done as per

190 Peterson et al. (2012) for the three AZA species at the Texas A\&M AgriLife Genomics core

191 facility using restriction enzymes SpeI and MboI (Koala and Addax) or SphI and MluCI

192 (IncaTern) for paired-end $150 \mathrm{bp}$ reads and sequenced on a portion of an Illumina NovaSeq 6000

193 lane. For all three AZA species, raw sequencing data were demultiplexed, filtered, and genotypes

194 were called using the bioinformatics pipelines STACKS v.2.0 and VCFTOOLS (Catchen et al.,

195 2013; Danecek et al., 2011). SNP genotypes for Addax and Inca Tern were generated using the

196 de novo pipeline in STACKS with the following parameters, respectively: $\mathrm{m}=3, \mathrm{M}=3, \mathrm{n}=0$,

197 min_maf $=0.02, \mathrm{r}=0.7$ and $\mathrm{m}=3, \mathrm{M}=3, \mathrm{n}=0, \mathrm{~min} \_\mathrm{maf}=0.02, \mathrm{r}=0.6$. Genotypes were

198 called for Koala using the reference pipeline using a reference genome (GenBank Accession:

199 GCA_002099425.1) with min_maf $=0.02$ and $\mathrm{r}=0.80$ parameters. Individuals were omitted

200 using VCFTOOLS based on maximum missingness thresholds, set to two settings to quantify

201 missing data impacts: low (10\%) and high (staggered at 40\% in Addax, $60 \%$ in Koala, and $80 \%$ 
202 in Inca Tern). Maximum values for the high missing datasets were chosen based on the

203 distribution of missing genomic data for each species.

204 These processing steps yielded 11 SNP datasets for comparison, including two datasets in

205 which GBS and WGS Kakī data were subset to the same consensus 25 individuals to allow direct

206 comparisons between SNP data generation approaches (Kakī GBS subset and Kakī WGS subset,

207 respectively; Table 1).

208 For each of the 11 datasets, we calculated six pairwise relatedness estimators to compare

209 estimates directly and to investigate their impact on downstream breeding recommendations:

210 Allele-Sharing (AS; Gutiérrez et al., 2005)), Kinship Genetic Distance (KGD; Dodds et al.,

211 2015)), Wang Maximum Likelihood (TrioML; Wang, 2002, 2011)), Queller and Goodnight ( $\mathrm{R}_{\mathrm{xy}}$;

212 Goodnight et al., 1999)), Kinship INference for Genome-wide association studies (KING-robust;

213 Waples et al., 2019), and $\mathrm{R}_{\mathrm{AB}}$ (Korneliussen \& Moltke, 2015). AS estimates (Gutiérrez et al.,

214 2005) were calculated in the program CASC (Ivy \& Putnam, 2019), subsampling 1,000 loci and

215 using 1,000 iterations. AS values are scaled differently than genomic-based kinships, so we

216 transformed AS values using a linear piecewise regression (hereafter Molecular Kinship,

217 MolKin). KGD estimates were calculated in R using Dodds et al. (2015). TrioML and Rxy

218 estimates were calculated in COANCESTRY (Wang, 2011), specifying unknown allele

219 frequencies and inbreeding. KING-robust and $\mathrm{R}_{\mathrm{AB}}$ estimators were estimated using NGSRelate

220 (Korneliussen \& Moltke, 2015).

221 In comparisons to known pedigree values, we only included individuals with known

222 pedigree information (i.e., those with kinship values > 0) from each species. Each pairwise

223 relatedness estimator was directly compared to known pedigree kinships (PedKin) and pedigree

224 relatedness (kinship/2; PedRel) and compared to all other genomic-based relatedness estimators 
225 using Pearson's correlation coefficients in R. Distributions of each relatedness estimator and the

226 pedigree kinship values were plotted using the density plots in R package ggplot2 (Wickham,

227 2011). Each distribution was compared to the pedigree kinship and pedigree relatedness

228 distributions using the Kolmogorov-Smirnov test in R (R function ks.test; Marsaglia et al.,

229 2003).

230 All genomic pairwise relatedness estimates, regardless if individuals had known pedigree

231 information, were imported into the genetics tab in PMx (Lacy et al., 2012). This was in part to

232 evaluate how pedigrees could be improved when incorporating genomic-based relatedness

233 values. We generated a rank of all the mean kinships in the population to compare breeding

234 recommendations across relatedness estimators. Low mean kinship ranks indicate individuals

235 with the fewest relatives in the population that are most valuable for breeding. All of the

236 relatedness estimators (MolKin, KGD, $\mathrm{R}_{\mathrm{xy}}$, TrioML, KING-robust, and $\mathrm{R}_{\mathrm{AB}}$ ) were imported

237 directly into PMx as relatedness values. $\mathrm{R}_{\mathrm{xy}}$, TrioML, KING-robust, and $\mathrm{R}_{\mathrm{AB}}$ do not generate

238 pairwise self-to-self estimates; we manually added values of 0.5 (the theoretical expected self-

239 self-relatedness value) where absent to allow PMx import. We compared mean kinship rank lists

240 (top 20 males and females) to each other and to the known pedigree kinships using Kendall's tau

241 correlations for ranked data ( $\mathrm{R}$ function kendall.tau).

242 Results

243 We generated six relatedness estimates for each of 11 genomic datasets (Table 1). All of

244 the relatedness estimator distributions overlap pedigree-based kinships (PedKin) except for AS,

245 which is on an inflated scale to the rest of the estimators (Figure 2). Transforming the AS values

246 (MolKin) brought them within the range of pedigree kinships. Often, the restricted variance we

247 observed in AS values was also observed in the transformed MolKin values; however, there are 
notable instances where the MolKin distribution almost entirely overlapped the distribution of

249 pedigree kinships (Koala 10 and Koala 60). Distributions for genomic relatedness estimators

250 TrioML and RAB are zero inflated because relatedness values truncate at 0 . This zero-inflated

251 skew was substantially reduced when using WGS compared to reduced representation methods.

252 WGS was able to provide relatedness values for relatively unrelated individuals whereas RRS

253 truncated those values at 0 , thereby lumping less related individuals as unrelated. The $\mathrm{R}_{\mathrm{xy}}$

254 distribution was skewed downward, lower than the pedigree kinship distribution. Though several

255 distributions are visually similar to the known pedigree kinship values, only one of the estimated

256 relatedness distributions was statistically similar: KING-robust in Koala 10.

The highest correlation values between genomic-based relatedness and known pedigree-

258 based kinship values were found in datasets with low missing data or high power, but we found

259 overall similar correlations values regardless of the level of missing data (Figure 3). Correlations

260 for a species were often higher for the low (10\%) missing dataset counterpart for Koala (Koala

261 10: $r=0.8-0.88$, Koala 60: $r=-0.038-0.82)$ and Inca Tern (Inca Tern 10: $r=0.63-0.84$, Inca

262 Tern 80: $\mathrm{r}=0.61-0.87)$. This is particularly prevalent when comparing the low correlation

263 values of KING-robust and $\mathrm{R}_{\mathrm{AB}}$ compared to the pedigree kinship values (0.025 -0.038,

264 respectively). Though, low correlation values in certain estimators (i.e., KING-robust) were not

265 consistently found in high missing genomic dataset, which could signal to another factor not

266 represented in our comparisons. Similarly, higher correlations were also found in WGS data $(\mathrm{r}=$

$2670.69-0.82)$ than in GBS $(r=0.31-0.72)$, which has considerably fewer loci and thus statistical

268 power $($ Kaki WGS $=68,144$ and GBS $=19,395)$. In general, datasets with more SNPs produced

269 estimates of relatedness with higher precision than datasets with smaller numbers of SNPs

270 (Figure 3). This pattern was observed when comparing WGS and GBS datasets in Kaki (WGS 
271 subset mean $\mathrm{r}=0.78$; GBS subset mean $\mathrm{r}=0.64$ ) but also when comparing ddRAD datasets

272 across species. For example, we observed a higher correlation between relatedness estimators

273 and known pedigree kinship for koala datasets with $>50,000$ SNPs $(r=0.68)$ than for addax

274 datasets with $<20,000$ SNPs $(r=0.56)$ holding missing data low (Figure 3$)$. Relatedness

275 estimates produced from datasets with less missing data were more correlated across estimators

276 (e.g., Koala 60 and Koala 10, Figure 3). That being said, there are a number of confounding

277 variables (e.g., pedigree accuracy, number of individuals, species genome size) across our

278 datasets that could affect the resulting relatedness values and be the underlying reason why there

279 are inconsistencies across datasets.

280 Kendall's tau correlations on mean-kinship rank data were low overall and varied widely

281 across species and datasets (Figure 4). There was a weak pattern in which species with more

282 complete pedigrees had more high correlation values than those with more incomplete pedigrees.

283 For instance, the Addax 10 dataset, with 13\% known pedigree, showed a much lower correlation

284 (average 0.06) between pedigree-based and genomic-based mean-kinship rank than determined

285 based on the pedigree or on empirical data than Kakī WGS (average correlation 0.19 with 56.6\%

286 of the pedigree known). However, this pattern did not hold across all species; Kākāriki Karaka in

287 which $99.5 \%$ of the pedigree was known prior, the average Kendall's tau correlation value was

288 0.10. In datasets with higher power, i.e., the WGS datasets, there are consistently higher

289 Kendall's tau correlation values among all estimators compared to those by reduced

290 representation methods. Datasets with less missing data echo this pattern of higher and more

291 consistent correlation values.

\section{Discussion}


294 genomic-based relatedness estimator for intensively managed conservation breeding populations

295 (Ivy et al., 2016); however, our results indicate that allele-sharing is be the least appropriate

296 estimator to improve conservation pedigrees. Allele-sharing values are on a vastly different scale

297 than pedigree kinships and frequency-based relatedness estimators. Because allele-sharing

298 provides a direct measure of identity-by-state, as opposed to a frequency-based estimate of

299 identity-by-descent, this estimator is expected to overestimate pairwise relatedness when genetic

300 diversity is low. This is salient for estimating relatedness in genetically-depauperate conservation

301 breeding programs, where the probability of identity-by-state is inflated (Henkel et al., 2012). As

302 such, any potential use of allele-sharing would require rescaling using a complex transformation

303 for incorporation into pedigree management, for example, the linear piecewise transformation

304 used here. However, transformations can introduce errors and reduce accuracy and precision of

305 relatedness estimates; in addition, a estimator that requires a complex transformation for use

306 suggests that it is ill fit for purpose. An essential quality of genomic relatedness measures for

307 conservation management is that there is sufficient variance to distinguish relationships. Our

308 results show that allele-sharing estimators and their transformed molecular kinships provide little

309 variance in either allele-sharing or the transformed molecular kinship values to provide the

310 necessary statistical power to confidently distinguish relationships. This inconsistency may be

311 influenced by the structure of the underlying pedigrees and each of their idiosyncrasies.

312 Regardless, for small populations of conservation concern, we would not recommend using

313 either allele-sharing or its derived molecular kinship due to the high inconsistency across

314 datasets. 
We anticipated that the inability to accurately estimate population allele frequencies in

316 captive breeding programs would lead to biased relatedness estimates when using frequency-

317 based estimators. However, we generally found high correspondence between relatedness

318 estimators and known pedigree values, especially in cases of low missing data, more fully

319 resolved pedigrees, and high-resolution genomic data (WGS and large numbers of SNPs). $\mathrm{R}_{\mathrm{xy}}$,

320 TrioML and $\mathrm{R}_{\mathrm{AB}}$ estimates were biased downward and consistently underestimated relatedness

321 across all datasets. The range of KGD and KING-robust estimates were the most consistent with

322 pedigree-based relatedness values, yet there was still some inconsistency relative to the known

323 pedigree values across the datasets. There was no clear pattern of bias in over-estimating (or

324 under-estimating) relatedness compared to the known pedigree values. While there is no single

325 optimal relatedness estimator as each has its advantages and caveats, we recommend that

326 researchers formally test candidate estimators, including KING-robust and KGD, for their

327 systems.

328 Missing data can occur in genomic datasets generated for captive populations for several

329 reasons. While proactive collection and banking of high-quality DNA samples for genomic

330 analyses is ideal to mitigate many of the limitations due to missing data, logistical and ethical

331 constraints in handling captive animals (i.e., minimizing handling and the associated stress and

332 risks) makes collection of high-quality and representative samples a challenge. These limitations

333 often compel researchers to rely on low-quality samples opportunistically taken from veterinary

334 samples including mammalian blood or plasma samples. Missing data can be the direct result of

335 suboptimal quality samples as genetic material is low yield and/or degraded (Graham et al.,

336 2015). A secondary effect of the opportunistic sampling is that sequencing is often done in

337 batches as samples become available through routine veterinary visits. However, batch effects 
338 are common and can be problematic, reducing the common loci among sequence batches and

339 subsequently increasing the amount of missing data in a genomic dataset (Leigh et al., 2018).

340 Thus, proactive sampling and storage of high quality samples will be important in reducing the

341 downstream complications than can arise from opportunistic sampling (i.e., missing data).

342 Conservation breeding managers can learn from ongoing efforts to establish proactive and

343 standardized sample collection by groups including AZA Frozen Zoo (e.g., Chemnick et al.,

344 2009), Cryo-Intiative (Comizzoli, 2014), Frozen Ark (Clarke, 2009).

There was a wide range of results in correlations among mean kinship rank data derived

346 from pedigree kinship and the genomic-based relatedness estimates. For pedigrees with little to

347 no information, the addition of genomic-based relatedness estimates replace unknown

348 relationship information (pedigree kinship $=0$ ) and subsequently change the mean kinship ranks

349 more than for known relationships. This is well illustrated with the Addax results for which the

350 pedigree was $13 \%$ known prior to relatedness estimates and $22.1 \%$ known after their

351 implementation. Though correlations between genomic-based relatedness estimators and

352 pedigree kinship were generally high (> 0.82; omitting the AS-derived molecular kinship),

353 differences in relatedness estimator distribution shape and range act as factor of discordance

354 among mean kinship rank. For instance, the variance for pedigree metrics was much wider for

355 the Kākāriki Karaka than for the other metrics, and the distributions were shaped quite

356 differently for genomic estimates (normal distribution) compared to those for the pedigree

357 (trimodal distribution). While the Kākāriki Karaka pedigree is almost complete, it is shallow and

358 therefore subject to outbreeding among the recent founders due to in-situ population structure

359 (Andrews, 2013; Galla et al., 2019, 2020), which could be the underlying cause behind

360 deviations with mean kinship rank. Nevertheless, across datasets, we optimistically found 
361 relatively high correspondence among estimators for mean kinship rank in species with little of

362 the pedigree known. We acknowledge that mean kinship is just one of many factors that inform

363 conservation breeding pairing recommendations, including other genetic metrics (e.g.,

364 inbreeding), demography at institutions, health, and behavior of individuals (Lacy et al., 2012).

365 Though resolving more relationships in a species pedigree using genomic-based estimates will

366 change mean kinship rankings, the new rankings will be utilizing more evidence and provide

367 more insight into the captive population. Subsequent breeding recommendations will improve

368 and captive population will benefit in the long-term.

369 There was variable performance of relatedness estimators across species, sequencing

370 method, degree of missing data, and pedigree knowness and depth. Thus, we advocate for

371 explicit evaluation of relatedness estimators for each new dataset and system. Researchers can

372 use our methods as a structured workflow to test the relative performance of relatedness

373 estimators against a set of known pedigree relationships for their specific system. The most

374 accurate estimates of relatedness are predicted to come from genomic datasets generated using

375 sequencing approaches that maximize statistical power (e.g., WGS) with nominal missing data

376 (e.g., high-quality samples, single batch sequencing). We recommend using WGS data over

377 RRS, when it is not cost- or computationally prohibitive. Our results also highlight potential

378 pitfalls in relatedness estimation. Firstly, allele-sharing is not recommended due to its low

379 variance, sensitivity to missing data, and large bias requiring a statistical transformation which

380 performs inconsistently and adds a potential source of error. Further, several estimators $\left(\mathrm{R}_{\mathrm{xy}}\right.$,

381 TrioML, and $\mathrm{R}_{\mathrm{AB}}$ ) showed systematic downward biases due to slightly differing ranges

382 compared to kinship or truncation of values to 0 , features that were designed specifically for

383 inbred populations (Korneliussen \& Moltke, 2015; Wang, 2011). As such, we cannot recommend 
AS, $R_{x y}$, TrioML, and $R_{A B}$ as candidate estimators to be used for small populations. Lastly, even a robust relatedness estimator cannot overcome sampling deficiencies, so we urge proactive sample collection across the breadth and depth of pedigrees to facilitate accurate reconstruction

387 of relationships among individuals to improve pedigree-based conservation management.

\section{Acknowledgments}

389 We are grateful for the continued support of Te Rūnanga o Ngāi Tahu, Te Ngāi Tūāhuriri

390 Rūnanga, Te Rūnanga o Arowhenua, Te Rūnanga o Waihao and Te Rūnanga o Moeraki. We

391 thank all members of the Kakī and Kākāriki Karaka Recovery Programmes for their ongoing

392 support. The Kakī and Kākāriki Karaka research was funded by the Ministry of Business,

393 Innovation and Employment Endeavour Fund (UOCX1602 awarded to TES), the Brian Mason

394 Scientific and Technical Trust (awarded to SJG and TES), and the Mohua Charitable Trust

395 (awarded to TES).We would like to thank the Institute of Museum and Library Services National

396 Leadership, Grant MG-30-15-0102-15 awarded to EKL. Support for SSH and SJG came from

397 University of Wisconsin-Milwaukee College of Letters and Sciences, and NSF EPSCoR RII

398 Track-2 award (OIA-1826801), respectively. Special thanks to the AZA institutions that supplied

399 samples: Buffalo Zoo, Brookfield Zoo, Dallas Zoo, Fossil Rim Wildlife Center, Frozen Zoo at

400 the San Diego Zoo Wildlife Alliance, Kansas City Zoo, Louisville Zoo, Cleveland Metroparks

401 Zoo, Omaha’s Henry Doorly Zoo and Aquarium, The Living Desert Zoo and Gardens, San

402 Diego Zoo, and Saint Louis Zoo. Lastly, thanks to J. Ivy, A. Putnam, and Association of Zoos

403 and Aquarium's Molecular Data for Population Management Scientific Advisory Group who

404 facilitated this collaboration.

405 Conflict of Interest Statement

406 There are no conflicts of interest to report. 


\section{Data availability}

408 A subset of the genomic data used in this project were previously derived from two culturally

409 significant species, namely Kakī/Black Stilt and Kākāriki Karaka/Orange-Fronted Parakeet

410 (Galla et al. 2019; Galla et al. 2020) and are currently stored on a password protected server

411 (http://www.ucconsert.org/data/). Kakī and kākāriki karaka are taonga (treasured) species. For

412 Māori (the Indigenous Peoples of Aotearoa New Zealand), all genomic data obtained from

413 taonga species have whakapapa (genealogy that includes people, plants and animals, mountains,

414 rivers and winds) and are therefore taonga in their own right (Collier-Robinson et al. 2019).

415 Thus, these data are tapu (sacred) and tikanga (customary practices, protocols, and ethics)

416 determine how people interact with it. To this end, the passwords for the genomic data in this

417 manuscript will be made available to researchers on the recommendation of the kaitiaki

418 (guardians) for the iwi (tribes) and hapū (subtribes) that affiliate with them. Having said this, if

419 one or more of the editors or reviewers would like access to these data during the review process,

420 we are able to provide time-sensitive passwords upon request.

421 For Addax, Koala and Inca Tern, species managed by the Association of Zoos and Aquarium, we

422 will make the genomic data available upon acceptance on dryad.

\section{Authors' Contributions}

424 SSH, SJG, EKL, and TES conceived the research ideas and designed the methodology. SSH and

425 SJG generated the genomic datasets. SSH analyzed the data. SSH and SJG led the writing of the

426 manuscript. EKL and TES supervised the analysis and interpretation of the research. All authors

427 contributed to the manuscript preparation and gave final approval for submission. 
Table 1. Summary information on each of the 11 genomic datasets used including: Number of individuals currently managed in the captive population (\# Inds Managed), percentage of the pedigree known prior to genomic data incorporation (\% Pedigree Known), average generation per individual with standard deviation (Pedigree Depth), type of genomic technique used (Type), the maximum missingness threshold used (Max Missing), number of individuals with SNP data in the dataset (\# Inds), number of SNP loci in the dataset (\#Loci), percentage of the pedigree known after genomic data incorporation (\% analytic known)

\begin{tabular}{ccccccccc}
\hline Species & $\begin{array}{c}\text { \# Living } \\
\text { Inds } \\
\text { Managed }\end{array}$ & $\begin{array}{c}\text { \% Pedigree } \\
\text { Known }\end{array}$ & $\begin{array}{c}\text { Pedigree } \\
\text { Depth }\end{array}$ & Type & Max Missing & \# Inds & $\begin{array}{c}\text { \# Loci } \\
\text { Known Analytic }\end{array}$ \\
\hline Addax & 518 & $13.0 \%$ & $6.3 \pm 3.2$ & ddRAD & $10 \%$ & 53 & 17184 & $22.1 \%$ \\
Addax & 518 & $13.0 \%$ & $6.3 \pm 3.2$ & ddRAD & $40 \%$ & 58 & 17184 & $22.3 \%$ \\
Inca Tern & 305 & $55.5 \%$ & $1.2 \pm 1.2$ & ddRAD & $10 \%$ & 72 & 38356 & $59.2 \%$ \\
Inca Tern & 305 & $55.5 \%$ & $1.2 \pm 1.2$ & ddRAD & $80 \%$ & 76 & 38356 & $59.3 \%$ \\
Koala & 50 & $36.0 \%$ & $3.7 \pm 1.4$ & ddRAD & $10 \%$ & 44 & 50875 & $48.0 \%$ \\
Koala & 50 & $36.0 \%$ & $3.7 \pm 1.4$ & ddRAD & $60 \%$ & 49 & 50875 & $49.0 \%$ \\
Kakīi & 533 & $56.6 \%$ & $2.6 \pm 1.4$ & WGS & $<1 \%$ & 36 & 68144 & $66.6 \%$ \\
Kakī & 533 & $56.6 \%$ & $2.6 \pm 1.4$ & GBS & $<1 \%$ & 82 & 19395 & $68.0 \%$ \\
Kākāriki Karaka & 184 & $99.5 \%$ & $2.5 \pm 0.7$ & WGS & $0 \%$ & 36 & 90949 & $99.5 \%$ \\
Kaki (subset) & 533 & $56.6 \%$ & $2.6 \pm 1.4$ & WGS & $<1 \%$ & 25 & 68144 & $65.0 \%$ \\
Kaki (subset) & 533 & $56.6 \%$ & $2.6 \pm 1.4$ & GBS & $<1 \%$ & 25 & 19395 & $65.0 \%$ \\
\hline
\end{tabular}




\section{Figure Captions}

Figure 1. Study species and key pedigree challenges faced by each conservation breeding program. Schematic pedigrees denote missing data with white circles/squares and existing data with black circles/squares.

Figure 2. Distribution density plots of each relatedness estimator. Please note that the x-axes vary across panels.

Figure 3. Heatmaps depicting the pairwise Pearson's correlation coefficient between relatedness estimators with darker gradient colors denoting higher correlation values. between sets of mean kinship rank derived from relatedness estimators. Above the gray diagonal 
References:

Andrews, B. J. (2013). Conservation Genetics of the orange-fronted kakariki (Cyanoramphus malherbi). University of Canterbury.

Ballou, J. D., \& Lacy, R. C. (1995). Identifying genetically important individuals for management of genetic variation in pedigreed populations. Population management for survival and recovery, 76-111.

Balloux, F., Amos, W., \& Coulson, T. (2004). Does heterozygosity estimate inbreeding in real populations? Molecular Ecology, 13(10), 3021-3031.

Bergner, L. M., Jamieson, I. G., \& Robertson, B. C. (2014). Combining genetic data to identify relatedness among founders in a genetically depauperate parrot, the Kakapo (Strigops habroptilus). Conservation Genetics, 15(5), 1013-1020.

Cassell, B. G., Adamec, V., \& Pearson, R. E. (2003). Effect of incomplete pedigrees on estimates of inbreeding and inbreeding depression for days to first service and summit milk yield in Holsteins and Jerseys. Journal of Dairy Science, 86(9), 2967-2976.

Catchen, J., Hohenlohe, P. A., Bassham, S., Amores, A., \& Cresko, W. A. (2013). Stacks: An analysis tool set for population genomics. Molecular Ecology, 22(11), 3124-3140.

Chemnick, L. G., Houck, M. L., \& Ryder, O. A. (2009). Banking of Genetic Resources. Conservation genetics in the age of genomics (pp. 124-130).

Clarke, A. G. (2009). The Frozen Ark Project: The role of zoos and aquariums in preserving the genetic material of threatened animals. International Zoo Yearbook, 43(1), 222-230. Retrieved June 9, 2021, from http://www.frozenark.org

Comizzoli, P. (2014). C-29: The Pan-Smithsonian cryo-initiative-freezing for the future. Cryobiology, 69(3), 509.

Danecek, P., Auton, A., Abecasis, G., Albers, C. A., Banks, E., DePristo, M. A., Handsaker, R. E., et al. (2011). The variant call format and VCFtools. Bioinformatics, 27(15), 2156-2158.

Dodds, K. G., McEwan, J. C., Brauning, R., Anderson, R. M., Stijn, T. C., Kristjánsson, T., \& Clarke, S. M. (2015). Construction of relatedness matrices using genotyping-by-sequencing data. BMC Genomics, 16(1), 1047.

Faust, L., Bergstrom, Y., Thompson, S., \& Bier, L. (2019). PopLink Version 2.5. Lincoln Park Zoo. Chicago, IL.

Fernández, J., \& Toro, M. A. (1999). The use of mathematical programming to control inbreeding in selection schemes. Journal of Animal Breeding and Genetics, 116(6), 447466.

Fernández, Jesús, Villanueva, B., Pong-Wong, R., \& Toro, M. Á. (2005). Efficiency of the use of pedigree and molecular marker information in conservation programs. Genetics, $170(3)$, 1313-1321.

Ferrie, G. (2017). Using Molecular Genetic and Demographic Tools to Improve Management of Ex Situ Avian Populations.

Flanagan, A. M., Masuda, B., Grueber, C. E., \& Sutton, J. T. (2021). Moving from trends to benchmarks by using regression tree analysis to find inbreeding thresholds in a critically endangered bird. Conservation Biology.

Flanagan, S. P., \& Jones, A. G. (2019). The future of parentage analysis: From microsatellites to SNPs and beyond. Molecular Ecology, 28(3), 544-567.

Galla, S. J., Forsdick, N. J., Brown, L., Hoeppner, M. P., Knapp, M., Maloney, R. F., Moraga, R., et al. (2019). Reference genomes from distantly related species can be used for discovery of single nucleotide polymorphisms to inform conservation management. Genes, 
10(1).

Galla, S. J., Moraga, R., Brown, L., Cleland, S., Hoeppner, M. P., Maloney, R. F., Richardson, A., et al. (2020). A comparison of pedigree, genetic and genomic estimates of relatedness for informing pairing decisions in two critically endangered birds: Implications for conservation breeding programmes worldwide. Evolutionary Applications, 13(5), 9911008.

Galla, S. J., Brown, L., Couch-Lewis, Y... Steeves, T. E. (Preprint). The relevance of pedigrees in the conservation genomics era. Authorea. doi:

Giglio, R. M., Ivy, J. A., Jones, L. C., \& Latch, E. K. (2016). Evaluation of alternative management strategies for maintenance of genetic variation in wildlife populations. Animal Conservation, 19(4), 380-390.

Giglio, Rachael M., Ivy, J. A., Jones, L. C., \& Latch, E. K. (2018). Pedigree-based genetic management improves bison conservation. Journal of Wildlife Management, 82(4), 766774.

Glaubitz, J. C., Casstevens, T. M., Lu, F., Harriman, J., Elshire, R. J., Sun, Q., \& Buckler, E. S. (2014). TASSEL-GBS: A High Capacity Genotyping by Sequencing Analysis Pipeline. PLoS ONE, 9(2), e90346.

Goodnight, K. F., Queller, D. C., \& Goodnight, K. F. (1999). Computer software for performing likelihood tests of pedigree relationship using genetic markers. Molecular Ecology, 8(7), 1231-1234.

Gooley, R., Hogg, C. J., Belov, K., \& Grueber, C. E. (2017). No evidence of inbreeding depression in a Tasmanian devil insurance population despite significant variation in inbreeding. Scientific Reports, 7(1).

Graham, C. F., Glenn, T. C., Mcarthur, A. G., Boreham, D. R., Kieran, T., Lance, S., Manzon, R. G., et al. (2015). Impacts of degraded DNA on restriction enzyme associated DNA sequencing (RADSeq). Molecular Ecology Resources, 15(6), 1304-1315.

Gutiérrez, J. P., Royo, L. J., Álvarez, I., \& Goyache, F. (2005). MolKin v2.0: A computer program for genetic analysis of populations using molecular coancestry information. Journal of Heredity, 96(6), 718-721.

Hammerly, S. C., de la Cerda, D. A., Bailey, H., \& Johnson, J. A. (2016). A pedigree gone bad: increased offspring survival after using DNA-based relatedness to minimize inbreeding in a captive population. Animal Conservation, 19(3), 296-303.

Hedrick, P. P. W., Lacy, R. C., \& Baker, C. S. (2015). Measuring relatedness between inbred individuals. Journal of Heredity, 106(1), 20-25.

Henkel, J. R., Jones, K. L., Hereford, S. G., Savoie, M. L., Leibo, S. P., \& Howard, J. J. (2012). Integrating microsatellite and pedigree analyses to facilitate the captive management of the endangered Mississippi sandhill crane (Grus canadensis pulla). Zoo Biology, 31(3), 322335.

Hogg, C. J., Wright, B., Morris, K. M., Lee, A. V., Ivy, J. A., Grueber, C. E., \& Belov, K. (2019). Founder relationships and conservation management: empirical kinships reveal the effect on breeding programmes when founders are assumed to be unrelated. Animal Conservation, 22(4), 348-361.

Ivy, J. A., \& Putnam, A. S. (2019). Calculate Allele Sharing Coefficients (CASC) (version 1.0). San Diego, CA, USA: San Deigo Zoo Global.

Ivy, J. A., Putnam, A. S., Navarro, A. Y., Gurr, J., \& Ryder, O. A. (2016). Applying SNPderived molecular coancestry estimates to captive breeding programs. Journal of Heredity, 
107(5), 403-412.

Jannink, J. L., Bink, M. C. A. M., \& Jansen, R. C. (2001). Using complex plant pedigrees to map valuable genes. Trends in Plant Science, 6(8), 337-342.

Jones, O. R., \& Wang, J. (2010). Molecular marker-based pedigrees for animal conservation biologists. Animal Conservation, 13(1), 26-34.

Korneliussen, T. S., \& Moltke, I. (2015). NgsRelate: A software tool for estimating pairwise relatedness from next-generation sequencing data. Bioinformatics, 31(24), 4009-4011.

Lacy, R C, \& Pollak, J. P. (2014). Vortex: A Stochastic Simulation of the Extinction Process. Manual. Version 9.99c.

Lacy, Robert C., Ballou, J. D., \& Pollak, J. P. (2012). PMx: Software package for demographic and genetic analysis and management of pedigreed populations. Methods in Ecology and Evolution, 3(2), 433-437.

Leigh, D. M., Lischer, H. E. L., Grossen, C., \& Keller, L. F. (2018). Batch effects in a multiyear sequencing study: False biological trends due to changes in read lengths. Molecular Ecology Resources, 18(4), 778-788.

Lemopoulos, A., Prokkola, J. M., Uusi-Heikkilä, S., Vasemägi, A., Huusko, A., Hyvärinen, P., Koljonen, M. L., et al. (2019). Comparing RADseq and microsatellites for estimating genetic diversity and relatedness - Implications for brown trout conservation. Ecology and Evolution, 9(4), 2106-2120.

Li, H., Handsaker, B., Wysoker, A., Fennell, T., Ruan, J., Homer, N., Marth, G., et al. (2009). The Sequence Alignment/Map format and SAMtools. Bioinformatics, 25(16), 2078-2079.

Marsaglia, G., Tsang, W. W., \& Wang, J. (2003). Evaluating Kolmogorov's distribution. Journal of Statistical Software, 8(1), 1-4.

Milligan, J. L., Davis, A. K., \& Altizer, S. M. (2003). Errors associated with using colored leg bands to identify wild birds. Journal of Field Ornithology, 74(2), 111-118.

Montgomery, M. E., Ballou, J. D., Nurthen, R. K., England, P. R., Briscoe, D. A., \& Frankham, R. (1997). Minimizing kinship in captive breeding programs. Zoo Biology, 16(5), 377-389.

Morin, P. A., Martien, K. K., \& Taylor, B. L. (2009). Assessing statistical power of SNPs for population structure and conservation studies. Molecular Ecology Resources, 9(1), 66-73.

Mucha, S., \& Windig, J. J. (2009). Effects of incomplete pedigree on genetic management of the Dutch Landrace goat. Journal of Animal Breeding and Genetics, 126(3), 250-256.

Ott, J. (1974). Estimation of the recombination fraction in human pedigrees: efficient computation of the likelihood for human linkage studies. American Journal of Human Genetics, 26(5), 588-597.

Overbeek, A., Galla, S. J., Brown, L., Cleland, S., Thyne, C., Maloney, R., \& Steeves, T. E. (2020). Pedigree validation using genetic markers in an intensively managed taonga species, the critically endangered kakī (Himantopus novaezelandiae). Notornis, 67(709-716).

Pemberton, J. M. (2008). Wild pedigrees: the way forward. Proceedings of the Royal Society B: Biological Sciences, 275, 613-621.

Peterson, B. K., Weber, J. N., Kay, E. H., Fisher, H. S., \& Hoekstra, H. E. (2012). protocol to ddRAD. PLOS ONE.

Putnam, A. S., \& Ivy, J. A. (2014). Kinship-based management strategies for captive breeding programs when pedigrees are unknown or uncertain. Journal of Heredity, 105(3), 303-311.

Robinson, S. P., Simmons, L. W., \& Kennington, W. J. (2013). Estimating relatedness and inbreeding using molecular markers and pedigrees: the effect of demographic history. Moleculary Ecology, 22, 5779-5792. 
Rudnick, J. A., \& Lacy, R. C. (2008). The impact of assumptions about founder relationships on the effectiveness of captive breeding strategies. Conservation Genetics, 9(6), 1439-1450.

Santure, A. W., Stapley, J., Ball, A. D., Birkhead, T. R., Burke, T., \& Slate, J. (2010). On the use of large marker panels to estimate inbreeding and relatedness: Empirical and simulation studies of a pedigreed zebra finch population typed at 771 SNPs. Molecular Ecology, 19(7), 1439-1451.

Skare, Ø., Sheehan, N., \& Egeland, T. (2009). Identification of distant family relationships. Bioinformatics, 25(18), 2376-2382.

Smouse, P. E. (2010). How many SNPs are enough? Molecular Ecology.

Sonesson, A. K., \& Meuwissen, T. H. E. (2001). Minimization of rate of inbreeding for small populations with overlapping generations. Genetical Research, 77(3), 285-292.

Spielman, D., Brook, B. W., Briscoe, D. A., \& Frankham, R. (2004). Does inbreeding and loss of genetic diversity decrease disease resistance? Conservation Genetics, 5(4), 439-448.

Spielman, D., \& Frankham, R. (1992). Modeling problems in conservation genetics using captive Drosophila populations: Improvement of reproductive fitness due to immigration of one individual into small partially inbred populations. Zoo Biology, 11(5), 343-351.

Sun, M., Jobling, M. A., Taliun, D., Pramstaller, P. P., Egeland, T., \& Sheehan, N. A. (2016). On the use of dense SNP marker data for the identification of distant relative pairs. Theoretical Population Biology, 107, 14-25.

Wang, J. (2007). Parentage and sibship exclusions: Higher statistical power with more family members. Heredity, 99(2), 205-217.

Wang, J. (2014). Marker-based estimates of relatedness and inbreeding coefficients: An assessment of current methods. Journal of Evolutionary Biology, 27(3), 518-530.

Wang, Jinliang. (2002). An estimator for pairwise relatedness using molecular markers. Genetics, 160(3), 1203-1215.

Wang, Jinliang. (2011). Coancestry: A program for simulating, estimating and analysing relatedness and inbreeding coefficients. Molecular Ecology Resources, 11(1), 141-145.

Waples, R. K., Albrechtsen, A., \& Moltke, I. (2019). Allele frequency-free inference of close familial relationships from genotypes or low-depth sequencing data. Molecular Ecology, 28(1), 35-48.

Weeks, A. R., Sgro, C. M., Young, A. G., Frankham, R., Mitchell, N. J., Miller, K. A., Byrne, M., et al. (2011). Assessing the benefits and risks of translocations in changing environments: A genetic perspective. Evolutionary Applications, 4(6), 709-725.

Wickham, H. (2011). ggplot2. Wiley Interdisciplinary Reviews: Computational Statistics, 3(2), $180-185$.

Wildt, D., Miller, P., Koepfli, K. P., Pukazhenthi, B., Palfrey, K., Livingston, G., Beetem, D., et al. (2019). Breeding Centers, Private Ranches, and Genomics for Creating Sustainable Wildlife Populations. BioScience.

Wolfe, B. A., Aguilar, R. F., Aguirre, A. A., Olsen, G. H., \& Blumer, E. S. (2012). Sorta situ: The new reality of management conditions for wildlife populations in the absence of "Wild" spaces. New Directions in Conservation Medicine: Applied Cases of Ecological Health.

Wright, S. (1922). Coefficients of Inbreeding and Relationship. The American Naturalist, 56(645), 330-338. 
bioRxiv preprint doi: https://doi.org/10.1101/2021.07.08.451704; this version posted July 10, 2021. The copyright holder for this preprint (which was not certified by peer(jeview) is the author/funder. All rights reserved. No reuse allowed without permission.
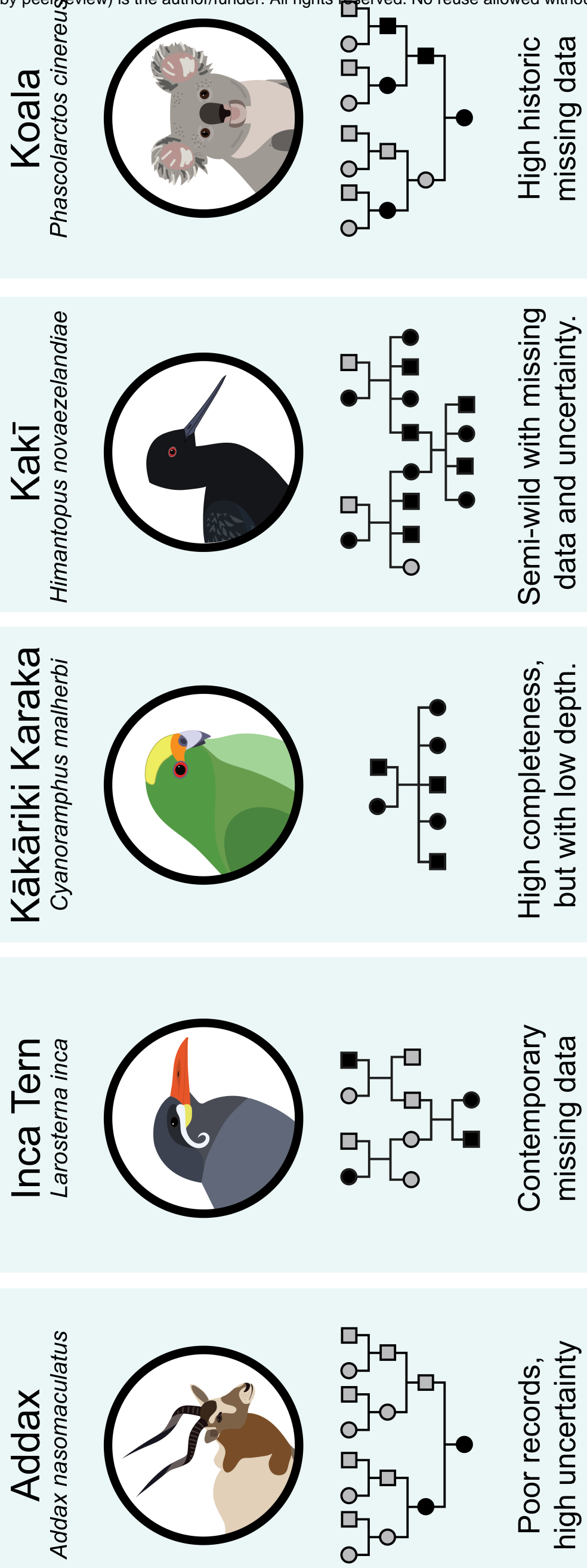


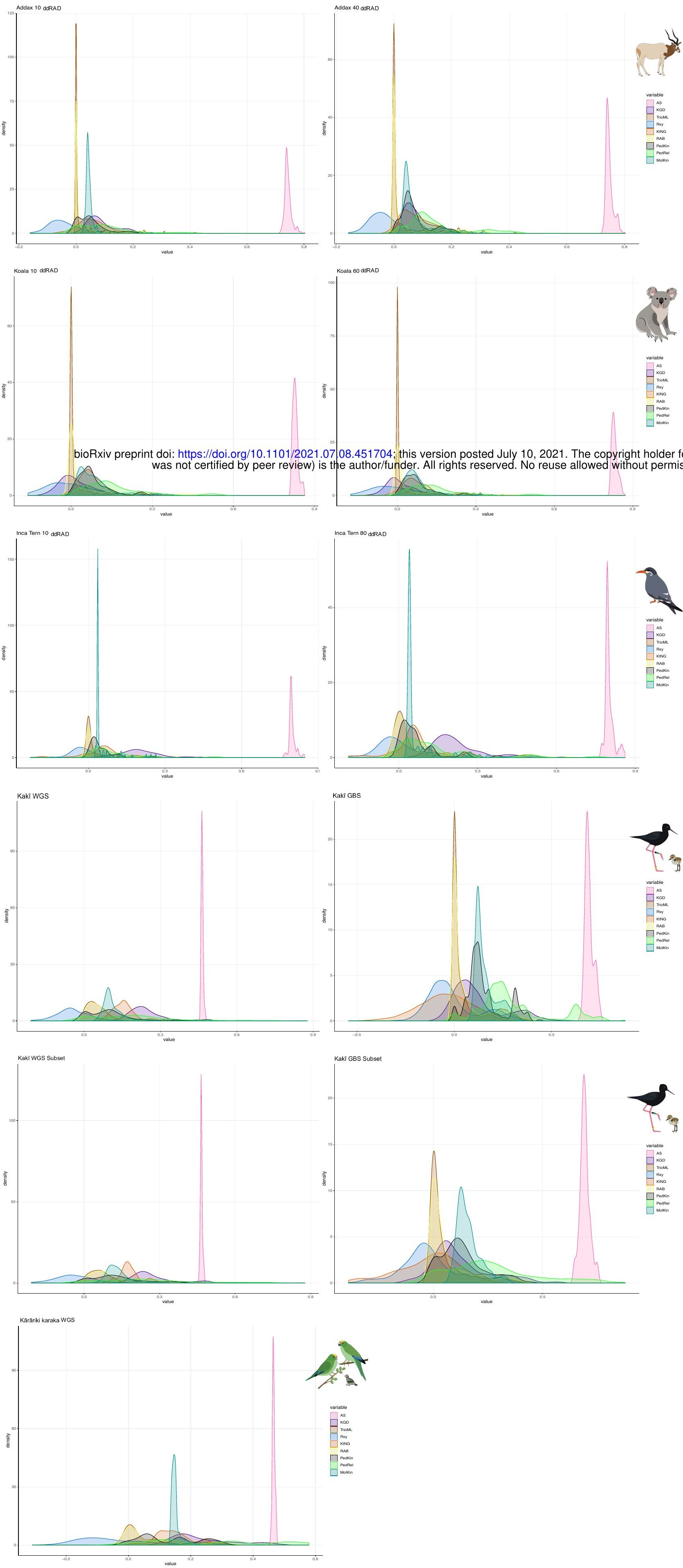




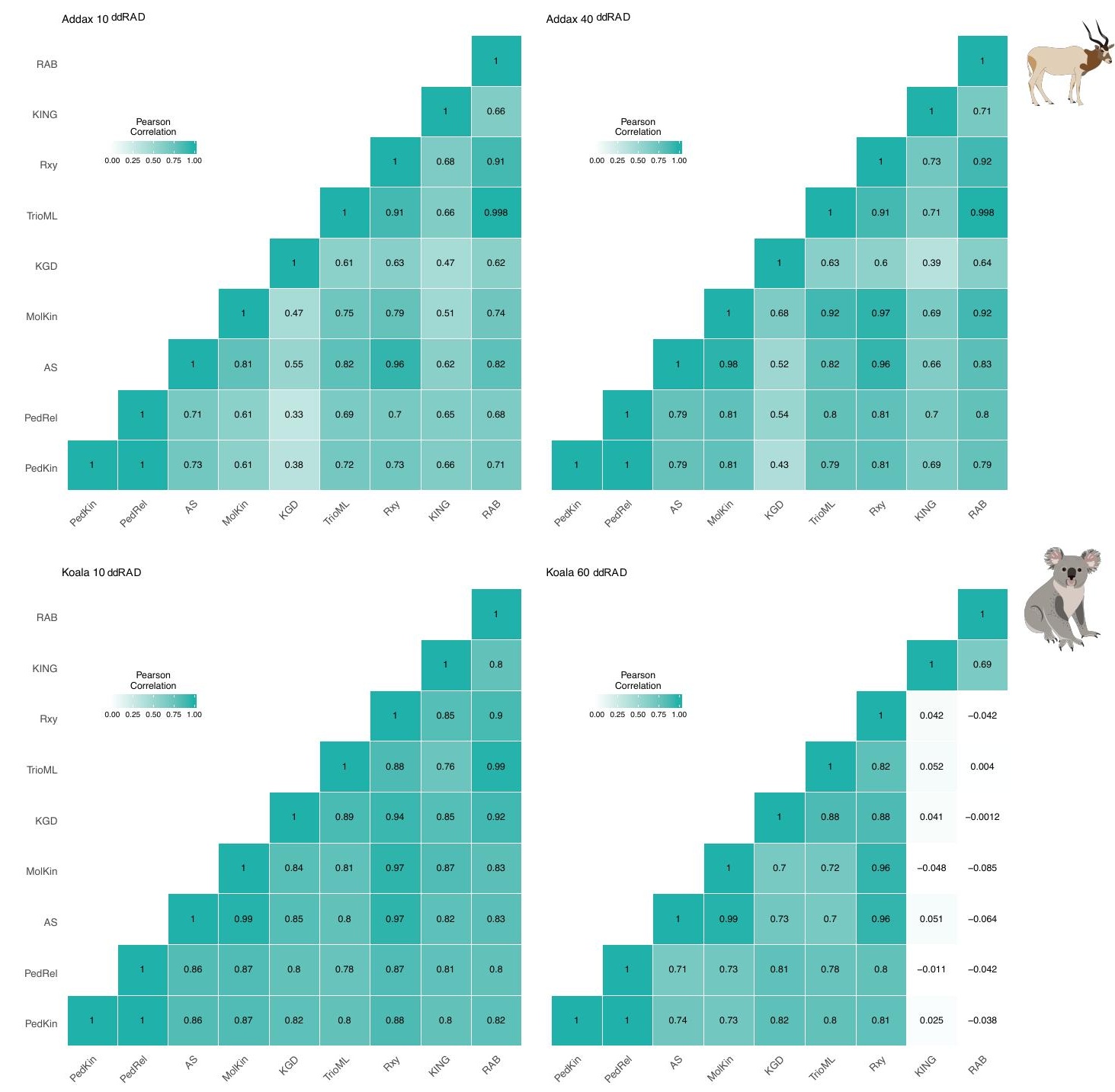

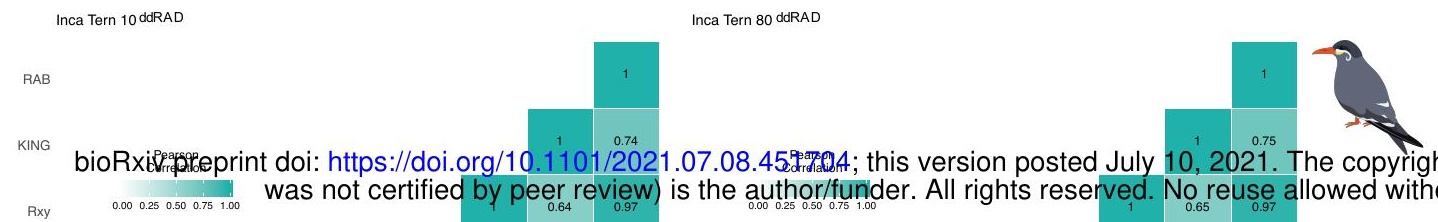
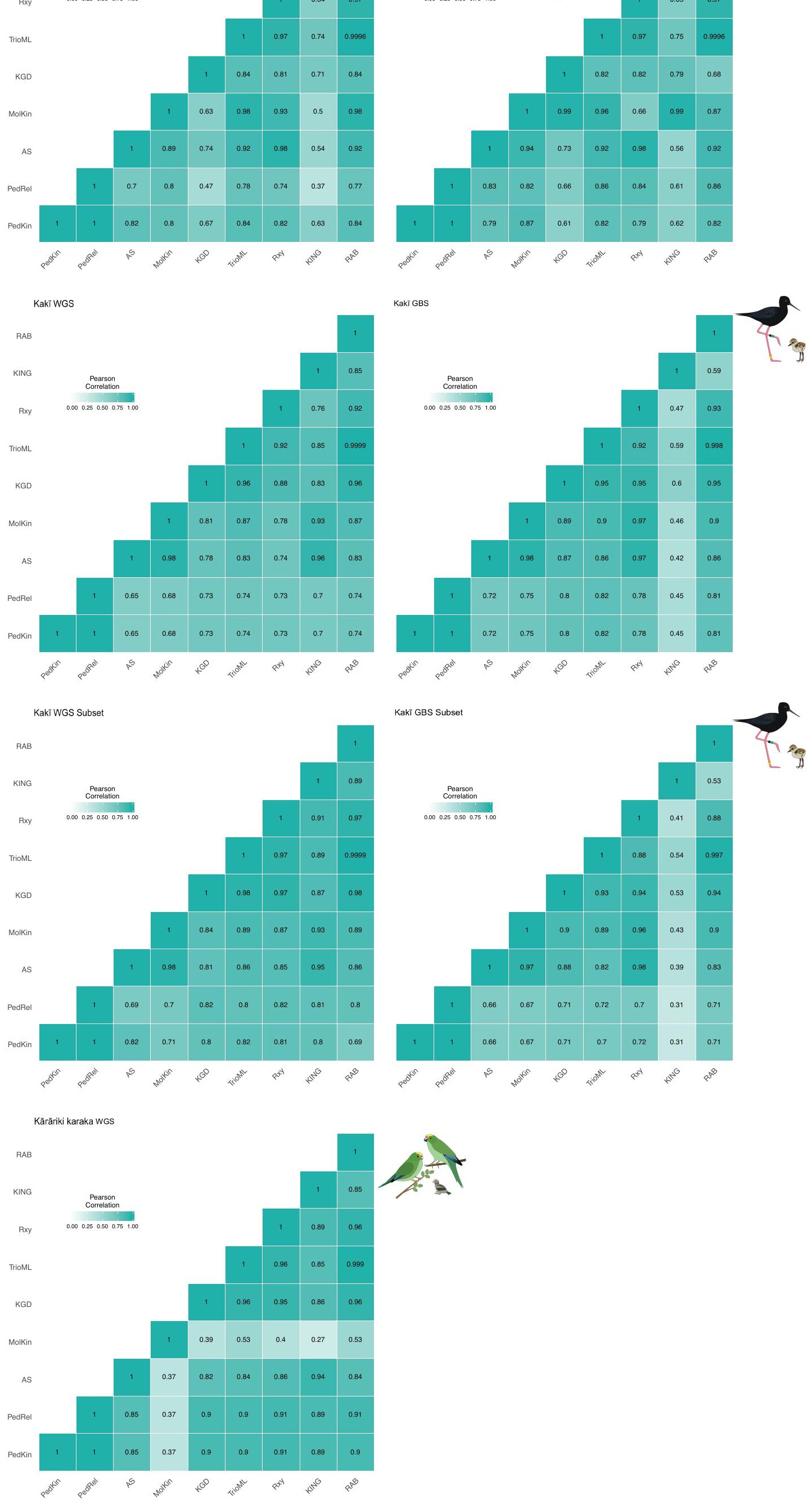

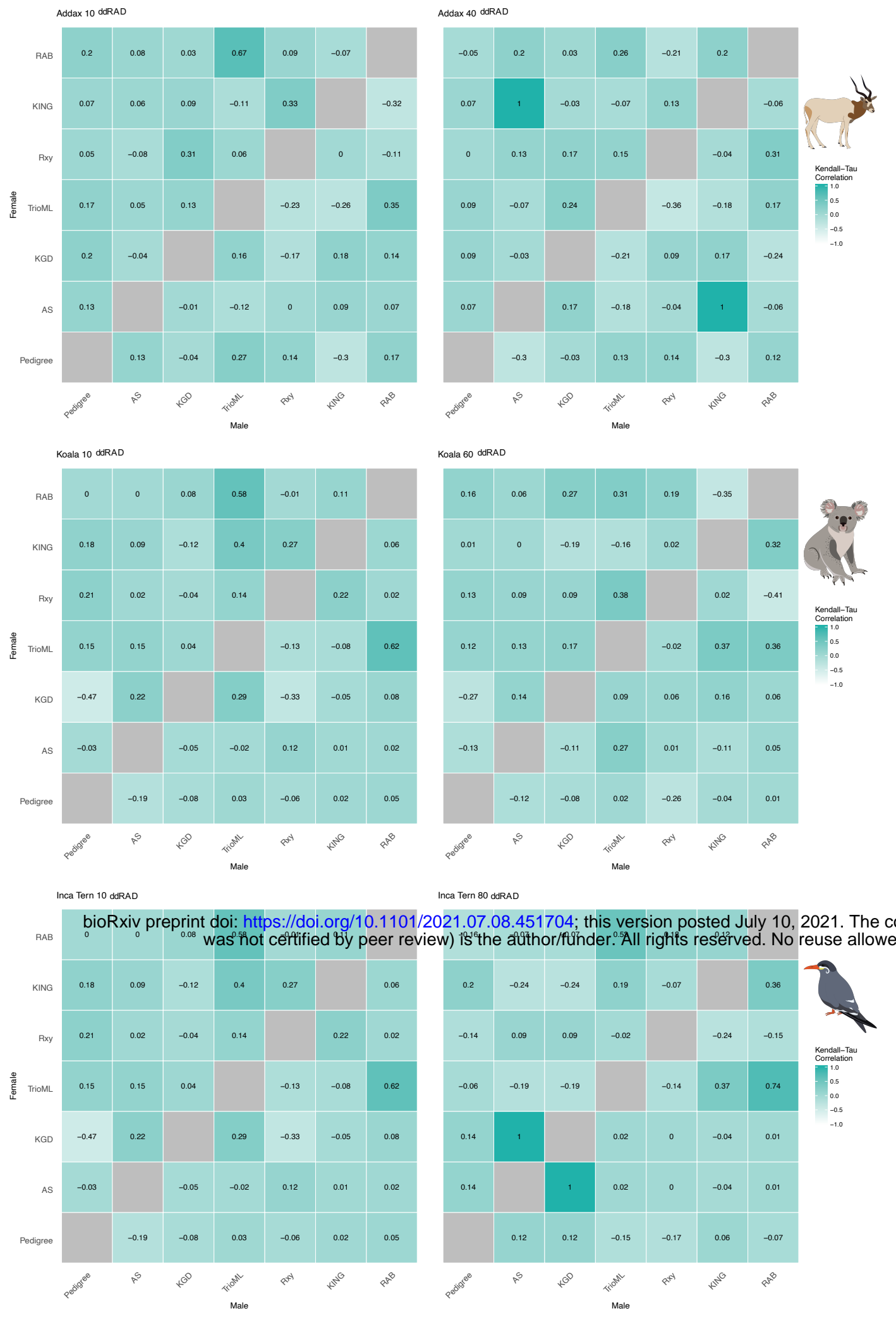

Inca Tern 80 doRAD

1704. this version posted July 10,2021 . The
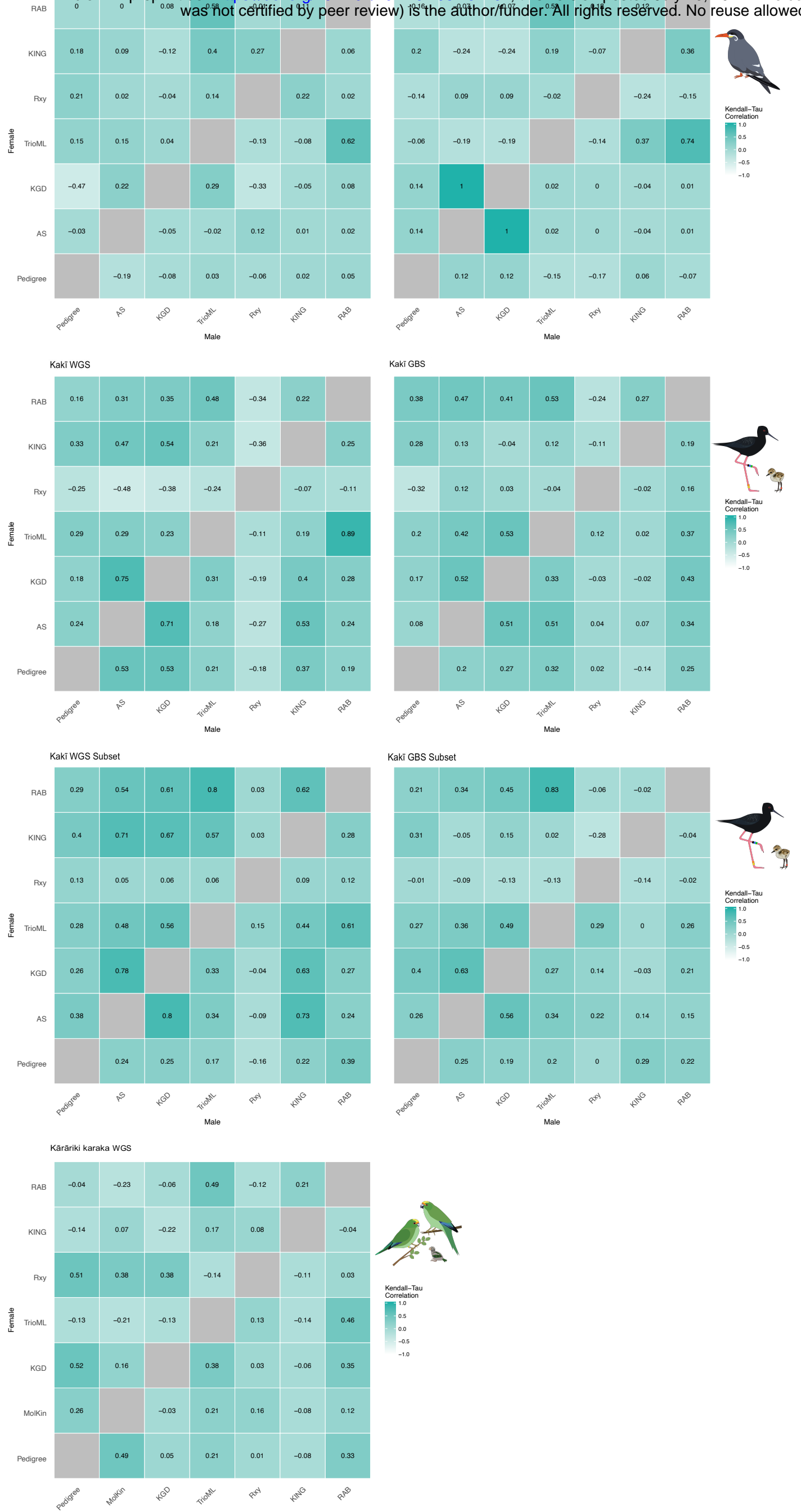